\title{
NEUROGÉNESIS Y ESTRUCTURA MODULAR DE LA CONCIENClA
}

NEUROGENESIS AND THE MODULAR STRUCTURE OF CONSCI OUSNESS

Resumen

Este artículo pretende dar cuenta de la génesis neurológica de nuestra subjetividad, esto es, del camino que recorremos hasta llegar a percibirnos a nosotros mismos como sujetos de nuestros actos y de nuestros pensamientos, construyendo una idea cada vez más extensa y precisa de nosotros mismos.

Consideramos que gran parte de los trabajos y de las teorías actuales sobre la conciencia chocan con problemas irresolubles porque carecen de esta perspectiva genética, olvidando que cuando nacemos no tenemos una subjetividad o conciencia. El artículo pretende así mostrar que es posible llegar a sensaciones subjetivas - sólo perceptibles y descriptibles en primera persona- a partir de fenómenos neurológicos impersonales, los cuales producen posteriormente el sujeto mental.

A lo largo del artículo tenemos en cuenta tanto los procesos psicológicos que están en la base de la conciencia como los sistemas neurológicos implicados en ellos. La conciencia aparece así como resultado de la coordinación unitaria de diversos sistemas o módulos neurológicos.

Palabras clave: consciencia, subjetividad, «yo», neurogénesis.
Abstract

This paper aims to explain the neurological genesis of our subjectivity, the path we follow until we can see ourselves as subjects of our acts and thoughts, forming an increasingly more accurate and extensive idea of ourselves.

We consider that a lot of current work and theories on consciousness come up against insoluble problems because they do not have this genetic view, forgetting that when we are born we do not have any subjectivity or consciousness. This article therefore intends to show that it is possible to reach subjective feelings - perceptible and describable only in the first person- from neurological impersonal phenomena which later produce the mental subject.

Throughout the article we deal with both psychological processes which form the basis of consciousness as well as the neuronal systems involved in them. In the end consciousness appears as a result of the unitary coordination of different neurological systems or modules.

Key words: Consciousness, subjectivity, self, neurogenesis. 


\section{NTRODUCCIÓN}

La conciencia incluye una serie de vivencias subjetivas que van desde la sensación, emoción y experiencia de un determinado momento hasta la reflexión sobre las causas y razones de la conducta y sobre la propia identidad. Pero un salto fundamental se produce cuando llegamos a la imputación unitaria de nuestras vivencias a un «yo». La creación de un sujeto mental y la reflexión sobre el mismo son hitos en la construcción del self, el cual incluye un pasado que condiciona un futuro de responsabilidades

Debemos abordar esta construcción como un largo proceso de unificación de sensaciones y experiencias, que finaliza en la constitución del sujeto mental, considerado como algo distinto del organismo que lo produce, aunque inseparable de él. Pensamos que la perspectiva genética que seguimos en este artículo ayudará a combatir el error metodológico (y conceptual) de muchos de los trabajos que se publican hoy sobre la conciencia: el de considerarla como una entidad que preexiste a las sensaciones y experiencias que percibe, en lugar de -invirtiendo los términos- concebirla y estudiarla como un producto de las mismas ${ }^{1}$. Algunos de los grandes modelos que se aventuran hoy sobre la conciencia, como los de Baars, Crick, Dennett, Edelman, Ornstein, etc. (1-8) mejorarían notablemente si incorporaran también la perspectiva ontogenética. Ella disuelve la mayor parte de los argumentos de teorías irreduccionistas, como las de Chalmers, Penrose, Searle (9-12), pues permite pensar que la experiencia de un determinado color o un cierto dolor, por ejemplo, antes de ser un fenómeno subjetivo, constituye un proceso objetivo o impersonal; y muestra que es razonable pensar que la subjetividad misma, o el sujeto psíquico - considerado como algo dentro del cuerpo- es el resultado de la coordinación de muchas experiencias, y de ninguna manera un sujeto que preexista a tales experiencias².

Cuando estudiamos empíricamente los fenómenos mentales, como es sabido, encontramos unas estructuras neurológicas que coinciden funcionalmente y específicamente con determinados estados de conciencia. Es lo que se ha denominado el «correlato neuronal» de la conciencia ${ }^{3}$. Mas esta localización no siempre resulta fácil cuando analizamos la estructura neurológica en su plenitud madurativa, es decir, cuando se ha llegado a una gran integración de muchas estructuras y funciones neuronales. Por ello creemos que el estudio evolutivo y la consideración de módulos permite mejorar el conocimiento de la correlación entre la neuroanatomía y los estados de conciencia, independientemente de cómo expliquemos esta correlación.

Nuestra orientación neurogenética en el estudio de la conciencia presta atención a las etapas de desarrollo neurológico y tiene en cuenta el análisis localizacionista de cada uno de los distintos módulos neurológicos que intervienen en la génesis de la conciencia. Esta perspectiva emergentista no contradice la vivencia posterior de la conciencia como un fenómeno unitario, ya que ésta es presentada como resultado final de un proceso unificador de conciencias parciales. A lo largo de este proceso se 
evidencia una correlación anatomo-funcional entre la actividad cerebral y los fenómenos mentales, la cual puede contribuir a un mejor planteamiento del problema de la conciencia.

Desde la perspectiva anatómica y funcional tomamos como punto de referencia de la localización neurológica los tres niveles o módulos clásicos, ordenados desde lo simple a lo complejo y de lo precoz a lo tardío desde el punto de vista evolutivo:

Sistema reticular de activación $(13,14)$ como sistema difuso y general de respuestas globales ante estímulos múltiples. Es un sistema de alerta general en el que se produce una unificación de la actividad nerviosa a partir de distintas aferencias.

- Sistema de activación reticular-talámico extendido, $\operatorname{ERTAS}(15,16,17)$, que añade el sistema talámico difuso $(18,19)$ al sistema anterior. La incorporación del tálamo implica una organización de las informaciones visuales, auditivas y somatosensoriales, tanto de las entradas como de las salidas sensoriales, de tal manera que se crea una interacción entre los distintos sistemas del tálamo y de éste con la corteza, a medida que se va produciendo el proceso de corticalización. - La incorporación al sistema anterior (ERTAS) de los sistemas corticales. En este modelo el punto fundamental es el núcleo reticular talámico que forma el nexo de una «matriz atencional» de la conciencia selectiva $(15,20)$.

\section{NI VELES EVOLUTIVOS ANATOMO-FUNCI ONALES DE LA CONCI ENCI A}

Abordamos pues la descripción de la evolución neurológica teniendo en cuenta su importancia en la producción del fenómeno de la conciencia, entendida ésta como experiencia personal y subjetiva y como reflexión constructora del «yo».

- El cerebro interno: la herencia animal.

En el momento del nacimiento disponemos de partes del sistema nervioso que podemos considerar muy maduros y por ello ya funcionales. Los sistemas de información y ejecución (los periféricos) son las zonas medulares y tronco-encefálicas, donde se producen los reflejos y reacciones ( reflejos tónico-cervicales, reflejos vestibulares, reacción de Moro, etc...) y cuyo centro de procesamiento (en el sentido de generador de respuestas específicas) es el sistema límbico (cerebro interno).

Los sistemas de información-ejecución del cerebro interno producen respuestas motoras rígidas y simples ante diversos estímulos. En estos casos la acción viene determinada por un estímulo sin mediación, habiendo una pre-determinación a ella. Las respuestas del niño son limitadas y previsibles, en condiciones normales. En este momento el sistema visual y auditivo tienen muy poca capacidad de discriminación y de orientación ante los estímulos del medio. Por ello los estímulos exteroceptivos (tacto, presión. temperatura) y los propioceptivos (posicionales en un sentido am- 
plio y determinados por la acción de la gravedad) son los que provocan las respuestas más primitivas del ser humano.

El sistema determinante de la orientación primaria es el cerebro interno, que desde los primeros momentos vitales se dirige hacia objetivos propios de la especie, de carácter innato e inespecífico, pero pronto de carácter biográfico al tener una transacción con el medio y una selección progresiva de las fuentes de refuerzo (objetos, situaciones y personas). La actividad del cerebro interno (sistema límbico, circuito de Papez), presente desde el nacimiento, controla las funciones relacionadas con nuestra herencia animal:

1. Instintos. Es necesario recordar que los instintos son buscadores de objetos en el medio y esta búsqueda de objetos es importante para el adiestramiento sensorial del niño, al tener éste desde el primer momento una imantación hacia el mundo de los objetos (mundo exterior a interiorizar en el campo de la conciencia). Este proceso de interacción del niño con el medio, junto con la determinación genética de la estructura neurobiológica que lo posibilita, produce una progresiva discriminación y orientación precisa hacia los objetos del mundo exterior (a los 4 ó 5 meses comienza la presión voluntaria de los objetos cuando se le ponen en las manos y a los 5 ó 6 meses dirige él la mano hacia los objetos) y hacia el propio cuerpo (a los 3 meses comienza a mirarse las manos). El resultado es una experiencia del mundo a través del cuerpo y del cuerpo a través de la experiencia sensitiva, sensorial y emocional (por este orden evolutivo) proporcionada por el mundo y memorizada gracias a los sistemas de recompensa tronco-encefálicos.

2. Memoria (en la que quedan relacionadas las situaciones y las emociones). La amígdala y el hipocampo, parte del cerebro interno, archivan en la memoria, cada vez con más precisión, los objetos, las personas y las situaciones. La memoria va unida a los aspectos emocionales del momento 0 momentos que rodearon la experiencia. Esta memoria emocional (en relación con el complejo amigdalino) es la memoria biográfica más elemental, que originará una situación interna de euforia y disforia ante un determinado objeto o situación. Se presenta paralelamente a la memoria de los objetos situados de manera discontinua ante nuestros sentidos, la cual es más dependiente del hipocampo.

3. Analizador rítmico. El funcionamiento de un analizador rítmico desde el nacimiento tiene una serie de consecuencias importantes en la génesis de la conciencia:

Determina el inicio del proceso comunicativo madre-hijo por ser el ritmo el modulador del diálogo tónico-afectivo $(21,22)$. Estableciendo el inicio de la futura dicotomía emisor-receptor, necesaria para la estructuración y conciencia del yo. El ritmo de mecimiento y modulación de los contactos madrehijo pone en sintonía el estado tónico y emocional de ambos, constituyendo la comunicación externa más primitiva. 
Al mismo tiempo, permite el agrupamiento de los elementos físicos-acústicos, por ejemplo- según ciertos elementos críticos (en relación con el tiempo de emisión de voz, con la variación de frecuencia del segundo formante, etc...), desde los primeros meses de la vida (23), preparando la división del mundo sensorial para la futura asignación de símbolos que organice el mundo exterior primero e interior después. En consecuencia, hay una categorización de los hechos acústicos de los fonemas, que posteriormente tendrán una asignación simbólica.

Como resultado de todo ello, podemos considerar la actividad del cerebro interno como el inicio de la diferenciación entre el cuerpo y el mundo exterior.

El conjunto de influencias externas sobre el niño en los tres primeros meses de vida. es de tipo protopático, global y generalmente vivido como situaciones dicotómicas. Situaciones que aparecen alternativamente: pregnancia-rechazo del objeto motrizmente, reposo-movimiento perceptivamente, placer-displacer emocionalmente. De estas dicotomías de las experiencias primitivas se evolucionará hacia experiencias progresivamente cada vez más diferenciadas. La memoria de estas experiencias y especialmente el alcance de un mayor grado de discriminación de las mismas dependerá en gran parte de la vivencia de los contrastes de manera alternativa.

Desde el punto de vista neurológico-evolutivo en el primer mes de vida se encuentran ya en un buen estado de maduración los elementos exteroceptivos y propioceptivos (relacionados con los aspectos posturales, principalmente con las reacciones de equilibrio y enderezamiento controladas por el sistema vestibular). En el segundo y tercer mes maduran de manera notable las conexiones vestibulares con los pares motores oculares. De estos hechos biológicos podemos inferir que la actividad propioceptiva (especialmente vestibular) determina una futura buena función sensorial (especialmente visual). Es decir, que la experiencia corporal es un camino para una adecuada experiencia del mundo. En una etapa posterior la experiencia sensorial tomará como objeto el propio cuerpo, estructurándolo en imágenes (descubrimiento visual de la mano como objeto especial entre los 3 y 5 meses).

Esta experiencia propioceptiva (que comienza con la estimulación del aparato vestibular del niño por el de la madre a través del mecimiento ritmico) determinará una buena maduración de los núcleos motores oculares y, en consecuencia, una fijación y seguimiento visual adecuados para la futura percepción (memorizada junto con su contexto por el hipocampo) y valoración emocional del mundo (memorizada por la amígdala). En resumen, podemos decir que la experiencia propioceptiva ( condicionada al comienzo por el otro -la madre-) es el principio de la diferenciación entre el cuerpo y el mundo exterior interiorizado. 
- Tálamo: El aspecto sensorio - emocional de la conciencia.

Pasados los tres primeros meses los sistemas sensoriales visual, auditivo y propioceptivo incrementan la capacidad de discriminación con la diferenciación progresiva de objetos, personas y situaciones en relación con el propio cuerpo. Esta discriminación va a ir creando la diferenciación entre el «yo» y el «otro» a través de la repercusión de los elementos externos diferenciados en estados internos diferentes.

La llegada de estas sensibilidades al tálamo y la integración que allí se produce determina la entrada en funcionamiento del sistema de conciencia selectiva propio del núcleo talámico reticular del adulto. Esta conciencia selectiva es la base de la subjetivación de la experiencia. En este sentido podemos hablar del inicio de la conciencia como experiencia en este nivel talámico. El tálamo orienta la disposición general del niño hacia un determinado sistema sensorial, manteniendo la atención en él e inhibiendo el resto de sistemas sensoriales como medio de primar la entrada selectiva de un tipo de estímulo a la corteza cerebral. Pero el tálamo no es un mero medio de regulación y facilitación de las entradas sensoriales en la corteza cerebral, sino que es también un cerebro sensorial, creador del aspecto sensorio-emocional de la conciencia en el mismo momento de la experiencia.

- Sistema tálamo-cortical: el «yo» agente

Un comienzo de la disociación mental entre lo interno y lo externo viene dada por los fenómenos conocidos como persistencia motriz y como segmentación funcional motriz.

El niño de 7 u 8 meses es capaz de mantener un cubo en una mano mientras abre la otra para recibir un segundo cubo. Esta actividad es una muestra de la aparición de lo que podemos llamar «persistencia motriz» $(24,25)$ : el niño a partir de esta edad es capaz de emplear una parte de su cuerpo (una mano) en una actividad mientras otra parte (la otra mano) se emplea en otra. Pero la primera acción es una acción de mantenimiento, mientras que la segunda es una acción sobre la que se centra la atención perceptiva. La primera se produce gracias a la influencia talámica sobre la actividad cortical, estableciendo las conexiones tálamo-corticales que permiten mantener constante una actitud motriz preexistente (de manera automática) mientras la atención se emplea en otra tarea (de manera intencional). La atención del niño en este momento se nos muestra como un proceso específico, dirigido hacia un objeto externo, mientras se mantiene un control de la actividad corporal que es capaz de funcionar de manera disociada.

La segmentación funcional del cuerpo y la posibilidad de dirigir la atención hacia algunos objetos específicos externos establecen uno de los pilares iniciales de la diferenciación entre el «yo» y el «otro» como entidades independientes. Un sistema de mi cuerpo es capaz de realizar una actividad que demanda una total atención selectiva hacia la acción y los objetos involucrados (experiencia de alto nivel de 
conciencia) y otra parte mantiene, a la vez, otra acción previamente procesada sin necesidad de desplazamiento de la atención (experiencia de bajo nivel de conciencia).

El desplazamiento de la atención a diversos objetos sin necesidad de abandonar actividades básicas que se realizan simultáneamente crea progresivamente la vivencia de la experiencia de agente y posibilita la percepción de otros agentes como distintos de él.

- Sistemas corticales. El «yo» reflexivo.

Los sistemas corticales permiten mejorar el conocimiento del mundo de los objetos, especialmente relacionado con la corteza parietal, occipital y temporal. Por otra parte, los sistemas corticales de la región frontal nos introducen en el campo de la reflexión constituyéndose la conciencia como un objetivo en sí misma.

Al respecto, son importantes dos consideraciones generales. La primera, acerca de los objetos y de los proyectos de la conciencia. Pues en el segundo semestre de la vida, la imitación de la acción del cuidador, la provocación para que repita acciones interesantes anteriores, y la exploración del mundo que rodea al niño, determinan el aprendizaje de la permanencia del objeto (a los 8 meses los objetos pueden estar en la mente del niño aunque no estén a la vista, según la teoría de Piaget). Esta permanencia supone que el objeto mental se independiza progresivamente de las circunstancias temporales y espaciales concretas que rodean su percepción y memoria (a los 12 meses aproximadamente es evidente esta independencia) (26). A los 18 meses la capacidad de representación y el juego representativo permiten organizar en la mente no sólo las formas externas de los objetos sino también sus propiedades y funciones. Tras la representación -y ayudándola al mismo tiempo- se produce la simbolización, gracias sobre todo al lenguaje. El lenguaje viene a resaltar los objetos como una figura sobre un fondo, siguiendo el pensamiento de Vygotsky (27). La dimensión comunicativa va unida, a partir de los 2 años, a la función del lenguaje interno por la que la conducta ya no se rige por los estímulos externos sólo, sino que el lenguaje ya es capaz de controlar y analizar la conducta del niño. Gracias a la mediación del lenguaje se rompe la conducta regida por las reacciones estímulo-respuesta. La progresiva evolución desde la sensopercepción del objeto a la simbolización del mismo nos permitirá el paso de los objetos de la experiencia a los objetos de la reflexión.

La segunda consideración es que el pleno funcionamiento de los sistemas corticales va a posibilitar la reflexión sobre sí, característica de la conciencia humana. El inicio de la capacidad reflexiva, aproximadamente a los 3 años, aparece como consecuencia del establecimiento del lenguaje interno, creando una «interfase» que permite la observación de nuestras respuestas. Al aplicar lo verbal para observar y designar las vivencias externas e internas del niño comenzará la génesis de la conciencia reflexiva. La nitidez y diferenciación de tales observaciones, dirigidas desde el lóbulo frontal, depende del grado de desarrollo madurativo de los sistemas corticales restantes. 
Tras estas consideraciones generales podemos pasar al análisis detallado de los sistemas corticales:

1. Corteza parietal, occipital y temporal. Conciencia corporal y del espacio circundante. La región parietal recoge las terminaciones propioceptivas, que informan de las posturas y movimientos del niño, así como de las estimulaciones tactiles. La representación espacial (junto con la representación de los objetos y de los movimientos) depende de la región occipital-parietal. Con estos elementos, acompañados de un importante factor emocional, se va creando una imagen corporal de tipo figurativo muy relacionada -quizás complementándola- con la imagen percibida por el niño en el espejo, resaltada en su momento por Lacan.

Los trabajos de experimentación animal llevados a cabo por Hyvarinen y Poramen (28) nos refieren la existencia en la región parietal de columnas neuronales sensibles a estímulos propiceptivos relacionados con la movilización de la mano hacia un objeto, ante la visión del objeto, ante el estiramiento de la piel (análogo al que se produce en el lanzamiento del brazo) hacia el objetivo e incluso la posibilidad de neuronas integradoras (en el surco interparietal) que se estimulan ante cualquiera de los hechos antes citados, siempre y cuando los objetos (alimentos, por ejemplo) estén a una distancia que haga posible su captura, y que además exista una motivación para alcanzarlo (hambre, en ese caso).

Así pues, la integración neurológica de visión, propiocepción, exterocepción y motivación en relación con un objeto alcanzable es un sustrato anatomo-funcional de la conciencia corporal. Este sustrato podemos considerarlo como base de la conciencia del propio cuerpo como sujeto que realiza acciones dentro de un espacio circundante. De modo que el cuerpo en acción dentro de un espacio tiene su correlato anatomo-funcional básico en la región parietal.

A través de la actividad manipulativa viene a añadirse un conocimiento del espacio circundante. La motivación para la toma de los objetos, presente de manera especial en la primera infancia por la conducta estímulo-respuesta generada por el binomio medio-niño, es otro factor a añadir para completar las condiciones básicas indicadas por los trabajos de Hyvarinen y Poranen (28). Condiciones que de manera esquemática son:

Integración de los aspectos propioceptivos, exteroceptivos de la manipulación. Implica la maduración (mielinización) de las vías nerviosas de la propiocepción consciente que llegan a la región parietal media y de las vías exteroceptivas del tacto.

Integración de la visión del objeto a manipular. I mplica la maduración de los sistemas de información inconscientes de la dirección (copia eferente del reflejo de captura foveal, propiocepción de musculatura del cuello y tronco..) y de la distancia del objeto ( reflejo de acomodación del cristalino, paralaje binocular...) como sistema inconsciente de localización del objeto. 
Este sistema inconsciente organiza en el cerebelo los programas básicos de captura del objeto. Pero después de actuar este sistema de aproximación al objeto (a partir de los 20 semanas) aparece la parte consciente de la manipulación que es el ajuste terminal donde la mano se adapta al objeto guiada por la corteza occipito-parietal.

Una distancia que permita tomar el objeto. Los sistemas corticales de integración parietal en el animal de experimentación no se activan si el objeto visto no está al alcance de la mano, lo que implica una experiencia manipulativa que va a crear un ámbito de actuación que podemos llamar espacio circundante. En el niño la posibilidad de acceder a la conciencia de un espacio circundante podrá realizarse cuando la captura de los objetos se realice con precisión, lo que proporcionará una estabilidad a las experiencias permitiendo al niño conocer la posibilidad de éxito y fracaso en la captura. Progresivamente se produce una optimización de las condiciones del éxito (información neuro-oftalmológica inconsciente de la dirección y distancia del objeto) y la anulación de la actuación ante las condiciones de fracaso. De manera que las condiciones de éxito y las condiciones de fracaso marcan los límites de la conciencia del espacio circundante. La realización de la captura del objeto con precisión se produce en el niño a partir de los 7-8 meses cuando progresivamente va controlando el cerebelo las distancias y coordinación de los movimientos.

Tener motivación para la toma del objeto. Ya que los sistemas corticales de integración parietal en el animal de experimentación no se activan si no hay motivación.

En resumen, consideramos que todos estos aspectos son los requisitos necesarios para que se vaya creando en el niño la conciencia corporal y la conciencia del espacio circundante, unidas ambas de forma indisoluble.

Ahora bien, la conciencia corporal gana progresivamente en riqueza gracias a la actividad motriz del niño a través de tres factores:

La precisión y segmentación motriz: en el recién nacido las respuestas motrices son masivas, inespecíficas, al no estar matizadas por el objetivo. A lo largo de los primeros años estas respuestas se van haciendo cada vez más precisas y adaptadas al objetivo, interviniendo solo los segmentos musculares necesarios y de la manera más adecuada. Esta segmentación permite una información propioceptiva diferenciada en función de cada actividad motriz específica y así la corteza parietal va teniendo una representación del cuerpo cada vez más analítica y precisa.

La automatización: la unión temporal entre diversos actos motrices repetidos muchas veces y siempre en el mismo orden constituye un proceso de automatización que crea, en el caso de la actividad motriz, una cadena cinética. 
Estas cadenas cinéticas crean una representación funcional del cuerpo que se suma a la anatómica producida por la segmentación.

La praxis: facilitará que los distintos segmentos corporales adopten una posición estable en el espacio. La praxis más elemental es la praxis ideomotora o gesto simple. Este gesto simple y su desarrollo en los primeros años de vida nos lleva a generar un progreso en la representación diferenciada de cada uno de los segmentos corporales-motrices (espacio corporal) que intervienen en el control de los distintos planos espaciales (espacio circundante). Del manejo de los objetos mediante la sucesión de gestos surge el conocimiento de las funciones y propiedades adscritas a los mismos, es decir, la adquisición de un significado (según la teoría de Piaget) condicionado éste también por la influencia del lenguaje social (Vygotski y teorías construccionistas). La adaptación de los segmentos motrices y de las cadenas cinéticas a las características del objeto genera una conciencia del cuerpo como instrumento de exploración y dominio del medio.

Según J eannerod y col. $(29,30)$, la función de asignar el significado a un objeto (conciencia semántica) está en relación con la vía visual ventral: occipito-temporopremotora (frontal). La localización de los objetos en el espacio (conciencia espacial) se realiza en la vía occipito-parieto-premotora (frontal). La maduración cortical, que lleva al funcionamiento de estas dos vías diferenciadas, constituye el sustrato neurogénico de la conciencia discriminatoria de los objetos y de su posición espacial (diferencia entre «¿qué es el objeto?» y «¿dónde está dicho objeto?»

2. Corteza frontal. Lenguaje y pensamiento reflexivo.

La maduración de las áreas prefrontales va unida a la introyección del lenguaje que a partir de este momento posibilita (multiplica las posibilidades de regular) la regulación de la conducta. Es un proceso progresivo que comienza con la aparición de la función representativa y simbólica a partir de los 18 meses.

La mediación del lenguaje determina, como hemos indicado anteriormente, que la conducta del niño pase de ser un conjunto semiestructurado de reacciones estímulo-respuesta a estar controlada por el contenido de las órdenes. Éstas, que vienen dadas por los cuidadores y que son repetidas internamente por el niño, le permiten inhibir su tendencia a responder a los estímulos externos, así como activar nuevas conductas.

El lenguaje sirve primeramente como propulsor para la ejecución de la orden motriz, siendo todavía incapaz de frenar un movimiento en curso (por ejemplo, si el niño está poniéndose los pantalones y le decimos que se los quite, no puede obedecernos); después será capaz de controlar las conductas mediante la orden verbal, si ésta es coherente con la circunstancia. Por último, el lenguaje interno 
creará sistemas de control de la conducta completamente independientes de las características del estímulo (el niño será capaz de responder con dos golpes ante un estímulo luminoso breve y con un golpe ante un estímulo luminoso prolongado venciendo la inercia propia del estímulo).

La creación de estos sistemas de conducta implica una utilización del lenguaje interior como sistema de control y de generación de conductas adaptadas a nuestras motivaciones y voluntad. La toma de decisiones para que se imponga un impulso motivacional sobre los demás exige un proceso de reflexión o deliberación sobre los distintos programas posibles que son simulados mentalmente. En todo este conjunto de actuaciones el lenguaje interior es el que representa de manera adelantada las hipótesis y contrasta los resultados virtuales para llegar a la selección más adecuada. A través de la formulación de hipótesis, la programación y la deliberación se va creando en el niño un mundo de referencias internas que le obliga constantemente a compulsar cada uno de los elementos posibles (resultados parciales hipotéticos) con los deseados en su memoria.

La progresión en este ejercicio de anticipación de actuaciones (pro-yección) unido a la memoria de otras actuaciones (retro-yección) va creando la posibilidad de dirigir el pensamiento reflexivo (lenguaje interior) hacia el núcleo biográfico generador de estos procesos mentales.

La introyección del lenguaje permite regular no sólo la conducta, en el sentido directo e inmediato, dirigida hacia objetivos a corto plazo, si no también incorporar las normas de conducta (éticas, estéticas....) del grupo, regulando así la conducta mediata, diferida, que supone medios y objetivos a largo plazo. El lenguaje interno incorpora directrices generales de actuación y mantiene a lo largo del tiempo la tensión necesaria para la realización de tareas a largo plazo, permitiendo los actos voluntarios o pre-meditados.

En esta etapa evolutiva, la actividad prefrontal representa la voluntad que supera las instancias de la primitiva motivación (unida al sistema límbico y los sistemas de refuerzo). Todos estos hechos evolutivos determinan el desarrollo funcional de los procesos de inhibición (de la tendencia a responder directamente ante los estímulos inmediatos) en relación con la vía orbitofrontal-núcleo dorsal del tálamo-hipocampo. Simultáneamente se crea una atención expectante (gracias a los procesos del lenguaje interno) que se manifiesta por una activación neurofisiológica que se registra en el área prefrontal (las ondas de W. Gray Walter (31) y las pruebas de variación de contingente). Esta atención expectante crea un estado de polarización de la atención a la espera de la aparición de un acontecimiento previamente anunciado (tras el ruido de la llave en la cerradura, el niño suspende su actividad y espera la aparición del padre). La combinación de acciones se realiza flexiblemente gracias a las relaciones sintagmáticas propias del primer uso del lenguaje. 
Paralelamente, el lenguaje diferencia la figura del fondo, proporcionando una visión del mundo, pero también permitiendo pronto resaltar el sujeto agente del sujeto paciente, hecho de decisiva importancia en la génesis social del yo ${ }^{4}$. La regulación de conductas a corto y largo plazo según las normas dictadas por nuestro lenguaje interior conducen a la capacidad de reversibilidad y descentración según la terminología de Piaget, y esto sucede a los 6 ó 7 años. La capacidad para volver mentalmente al inicio de una conducta 0 de analizarla desde distintos puntos de vista determina la creación de hábitos de reflexión (uso de razón) que incluyen la reflexión sobre la propia persona como unidad incorporada responsablemente en la sociedad. La capacidad reflexiva que proporciona el lenguaje permite el análisis del yo actuante.

3. Conexión interhemisférica. La reflexión sobre el «Yo».

La mielinización del cuerpo calloso es un proceso largo que alcanza un gran desarrollo anatómico desde los 8 a los 11 años. Funcionalmente representa la transferencia de información entre los dos hemisferios. En relación con el tema que estamos tratando nos interesa saber que el lenguaje localizado en el hemisferio dominante (generalmente el izquierdo) puede actuar codificando los contenidos del hemisferio no dominante relacionados con el aspecto figurativo del espacio, esquema corporal, sensaciones de contenido emocional y en general el mundo de lo inefable. Cuando se llega a la edad de 11 años la posibilidad de utilizar la transferencia de distintos códigos nos introduce en el mundo de los metalenguajes y nos lleva a la formulación de hipótesis y teorías. Es el momento en que el niño se formula de manera explícita preguntas que podemos reducir a dos de los grandes interrogantes vitales: el «¿quién soy yo?» y el «¿qué será de mi?» El mundo del lenguaje organiza y hace coherente el conjunto de experiencias, activas y receptivas, vividas por los demás módulos. El módulo del lenguaje, a través del vocabulario, descripciones, narraciones e historias, creencias, hipótesis, teorías, etc. da sentido a la experiencia general y, en particular, a la reflexión sobre el «yo».

\section{UN MODELO INTEGRADOR Y MODULAR}

El estudio genético de determinados sistemas anatomo-funcionales nos permite realizar las siguientes consideraciones:

1. Existencia de una innata determinación (instintos) para el desarrollo de los procesos de análisis (analizador rítmico, por ejemplo) y memorización -en la amígdala y el hipocampo- de los objetos humanos y no humanos del entorno, con la consiguiente valoración (sistemas de refuerzo-recompensa), todo lo cual determina una experiencia biográfico-emocional. 
2. La diferenciación entre la experiencia de la acción atenta e intencional y el mantenimiento automático de la actividad en la persistencia motriz (como al tomar un objeto con una mano, mientras se mantiene otro objeto en la otra) se relaciona con la maduración de las conexiones tálamo-corticales y marca la diferenciación entre las actividades con experiencia consciente y las actividades automáticas no conscientes.

3. La selección del canal de atención idóneo para cada acción (visual, auditivo, etc..) por parte de las estructuras talámicas relaciona la experiencia con la motivación, aportando un aspecto subjetivo y biográfico en toda experiencia.

4. La corteza cerebral parietal, a partir de la experiencia exteroceptiva, propioceptiva y visual de la manipulación motivada de objetos, crea una conciencia unitaria del cuerpo, del espacio circundante y de la eficacia funcional. La segmentación motriz (diferenciación funcional de las partes actuantes), la realización de secuencias (automatización como unión temporal) y de praxis (como unión espacial de los segmentos motrices) crean una representación del cuerpo, a la vez unitaria (todo él interviene como una unidad propositivamente) y analítica (podemos centrarnos selectivamente en cada una de sus partes), actuando en el tiempo y en el espacio.

5. La corteza frontal, mediante la introyección del lenguaje, permite guiar el análisis reflexivo sobre el agente actuante (producto de las sensaciones organizadas y memorizadas en los módulos anteriores) de una manera descentrada, viéndolo desde fuera como un objeto de observación distinto biográfica y emocionalmente de los otros objetos.

6. La conexión interhemisférica multiplica las posibilidades para que el «yo» biográfico-emocional reflexione sobre sí mismo, se sitúe críticamente frente a otros y frente al universo, y se constituya en proyecto ante preguntas como ¿quién soy yo? 0 ¿qué voy a hacer?

Podemos decir que el «yo» se genera a partir de una rica experiencia que se va organizando. Consideramos que la conciencia tiene una estructura pluralista y modular, a la que contribuyen muchos sistemas o conciencias parciales (corporal, espacial...). Esta estructura modular de la conciencia se integra jerárquicamente, de tal manera que el módulo prefrontal constituye la cima evolutiva del «yo» biográfico y social coordinando las transferencias entre los dos hemisferios.

Por supuesto, no se debe pensar que tales capacidades permitan a la conciencia controlar toda nuestra conducta, ni que los planes que ella traza no vengan dictados por otras instancias. Tampoco debemos olvidar que, al unificar nuestra experiencia interna, el «yo» realiza continuamente montajes muy variados acerca de nosotros mismos.

Esta visión genética y modular de la conciencia, que presenta el sujeto psíquico como resultado de un largo trayecto de unificación, permite considerar muchas patologías de la personalidad como defectos de este proceso. 


\section{NOTAS}

1. Esta perspectiva genética, presente en algunos clásicos, como Dewey o Piaget, no ha logrado pasar del ámbito de la psicología evolutiva al de los actuales estudios sobre la conciencia. Salvo algunas excepciones como el artículo historiográfico de Dalton (32).

2. Conviene desligar el problema de la conciencia considerada en cuanto subjetividad -cuestión que queda modificada por la presente perspectiva genética- del otro problema que consiste en explicar cómo se producen los fenómenos mentales a partir de activaciones combinadas de neuronas. Pues podríamos encontrar una solución para el segundo problema - por ejemplo, considerando los procesos mentales como fenómenos neurológicos emergentes al modo de una melodía interpretada por una orquesta-, sin por ello haber resuelto el primer problema, el de la subjetividad, que implica o bien la preexistencia o bien la creación de un sujeto mental.

3. No usamos el término «correlato» en un sentido dualista (al modo de Chalmers), como si los dos polos correlacionados fuesen diferentes. Hablamos de correlato sin prejuzgar la identidad o la diferencia radical entre las dos cosas correlacionadas (lo mental y lo neuronal). Crick (3) - nada sospechoso de dualista- o J ackendoff (33) también emplean el término «correlato». Que los fenómenos mentales 0 psíquicos no sean meras correspondencias sino que se identifiquen con algún tipo de proceso neuronal emergente (ya se trate de las oscilaciones sincrónicas de $40 \mathrm{~Hz}$, ya de un campo electromagnético, ya de «melodías» neuronales o de cualquier otro) es hoy sólo una hipótesis, aunque esta línea de trabajo impulsa la mayor parte de las investigaciones científicas (34-39). Para otras informaciones sobre este concepto, ver el libro de Metzinger (40).

4. El papel del lenguaje en la construcción social del yo va a ser continuo a lo largo de nuestra vida. El vocabulario de una lengua, en un momento histórico y dentro de un ambiente cultural dado, organiza y da sentido a nuestra experiencia externa e interna; por medio del lenguaje recibimos las descripciones que los demás hacen de nosotros y las historias o narraciones que cuentan sobre nuestras personas, todo lo cual contribuye a formar nuestra propia autoimagen por el reflejo especular que los demás nos transmiten (41-45)

\section{REFERENCI AS BIBLIOGRÁFICAS}

1. Baars, B.J .: A Cognitive Theory of Consciousness, New York: Cambridge University Press, 1988.

2. Baars, B.J .: «In the theatre of consciousness: Global Workspace Theory, a rigorous scientific theory of consciousness», J ournal of Consciousness Studies, 1997, 4, 4, 292-309. 
3. Crick, F.: The Astonishing Hypothesis: The Scientific Search for the Soul, New York: Charles Scribner»s Sons, 1994.

4. Dennett, D.C.: Consciousness Explained, Little: Brown, 1991.

5. Edelman, G.M.: The Remembered Present: A Biological Theory of Conciousness, New York, Basic Book, 1989.

6. Edelman, G.M.: Bright Air, Brilliant Fire: On the Matter of the Mind, New York: Basic Books, 1992.

7. Ornstein, R.: The Psychology of Consciousness, New York: Viking, 1972.

8. Ornstein, R.: The Evolution of Consciousness. The origins of the way we think, New York: Simon \& Schuster, 1991.

9. Chalmers, D.: Toward a Theory of Consciousness, Oxford: Oxford Univ. Press, 1996.

10. Chalmers, D.: The Conscious Mind: In Search of a Fundamental Theory, Nueva York: Oxford Univ. Press, 1996.

11. Penrose, R.: The Emperor»s New Mind, Oxford, New York, Melbourne: Oxford University Press, 1989.

12. Searle, J.: The Rediscovery of Mind, Cambridge, MA, The MIT Press, 1992.

13. Magoun, H.W.: The Waking Brain, Springfield, III, Thomas, 1963.

14. Moruzzi, G.: «Reticular influences on the EEG», Electroencephalography and Clinical Neurophysiology, 1964, 16, 2-17.

15. Newman J . and Baars B.J.: «A neural attentional model for access to consciousness. A global Workspace perspective», Concepts in Neuroscience, 1993, 4 (2), 225-90.

16. Newman J .: «Putting the puzzle together: Part I», J ournal of Consciousness Studies, 1997, 4 (1), 47-66.

17. Newman J.: «Putting the puzzle together: Part II», Journal of Consciousness Studies, 1997, 4 (2), 100-21.

18. Jaspers, H.H.: «Unespecific talamocortical relation» in Handbook of Neurophysiology, I. ed. J. Field, H.W. Magoun and V.E. Hall, Washington, American Psycological Society, 1987.

19. Thompson, R.F.: Foundations of Physiological Psychology, New York, Harper and Row, 1967.

20. Newman. J.: «Review: Thalamic contributions to attention and consciousness», Consciousness and Cognition, 1995, 4(2), 172-93.

21. Wallon, H.: Les origines du caractère chez leenfant, Paris, PUF, 1976.

22. Gómez Tolón, J .: Trastornos de la adquisición del lenguaje. Valoración y tratamiento, Madrid: Escuela Española, 1987.

23. Eimas P.D., "Linguistic processing of speech by young infants», Schiefelbusch and Lloyd, 1974, 55-73.

24. Fisher, M.: "Left hemiplejia and motor persistence», J. Nerv. Dis. 1956, 123, 201-18.

25. Gómez Tolón, J.: «Concepto de impersistencia motora en el hemipléjico y su valoración práctica en rehabilitación», Rehabilitación, 1974, 8 (4), 607-15. 
26. Piaget J.: La formation du symbole chez I»enfant, Paris, Delachaux et Niestlé, 1945.

27. Vigotsky, L.: El desarrollo de los procesos psicobiológicos superiores, Barcelona, Crítica, 1979.

28. Hyvarinen, J., Poranen, A.: «Function of the parietal associative area 7 as revealed from cellular discharges in alert monkeys», Brain, 1974, 97, 673-92.

29. J eannerod M.: «Mental imagery in the motor context», Neuropsychologia, 1995, $33,1419-1432$.

30. Jeannerod, M., and J. Decety.: «Mental motor imagery: a window into the representational stages of actino», Current Opinion in Neurobiology 1995, 5, 727-732.

31. Walter, W. G.: «Slow potential changes in the human brain associated with expectancy decision and intention», Electroencephalogr. Clin. Neurophysiol, 1967, 26, suppl., 123-30.

32. Dalton, T.C.: «The ontogeny of Consciousness: J ohn Dewey and Myrte McGraw»s contribution to a science of mind», J ournal of Consciousness Studies, 1999, 6 (10), 3-26.

33. Jackendoff, R.: Consciousness and the Computational Mind, Cambridge, Bradford Books / The MIT Press, 1987.

34. Makarenko V. and Llinas R.: «Experimentally determined chaotic phase synchronization in a neuronal system», Proceedings of the National Academy of Sciences of the United States of America ,1998, Dec 22;95(26), 15747-52.

35. Llinas R. y otros: «The neuronal basis for consciousness», Philosophical Transactions of the Royal Society of London - Series B: Biological Sciences, 1998, Nov, 29, 353(1377), 1841-9.

36. Llinas R and Ribary U.: «Consciousness and the brain. The thalamocortical dialogue in health and disease», Annals of the New York Academy of Sciences, 2001 Apr; 929, 166-75.

37. Carreras, A.: «Melodías neuronales», Actas del III Congreso de la S. L.M.F.C.E., San Sebastián, Ibaeta Filosofía, 2000, pp. 123-133.

38. McFadden, Johnjoe: «The conscious electromagnetic information (Cemi) field theory: The hard problem made easy», J ournal of Consciousness Studies, 2002, vol., 9, no 8, 45-60.

39. Pockett, S.: The Nature of Consciousness. A Hypothesis, Lincoln, NE, Writers Club Press, 2000.

40. Metzinger, T. ed.: Neural Correlates of Consciousness. Empirical and Conceptual Questions, Cambridge, MA, MIT Press, 2000.

41. Cooley, Charles H.: Human Nature and the Social Order, New York, Scribner»s, 1902.

42. Gergen, K.J .: The Saturated Self, Dilemmas of I dentity in Contemporary Life, New York, Basic Books, 1991. 
43. Burns, T.R. and Engdahl, E.: "The social construction of consciousness. part I: Collective consciousness and its socio-cultural foundations», Journal of Consciousness Studies, 1998, 5, 1, 67-85.

44. Burns, T.R. and Engdahl, E.: "The social construction of consciousness, part 2: Individual selves, self-awareness and reflectivity», J ournal of Consciousness Studies, 1998, 5, 2, 166-84.

45. Carreras, A. (ed.): Tras la Consciencia, Zaragoza, Mira/SIUZ, 1999.

J esús Gómez Tolón

Médico rehabilitador

Departamento de Fisiatría y Enfermería

Escuela Universitaria de Ciencias de la Salud

Universidad de Zaragoza

E-mail: jes@unizar.es

Alberto Carreras Gargallo

Filósofo y Psicólogo

Departamento de Filosofía

Facultad de Filosofía y Letras

Universidad de Zaragoza

E-mail: acaras@unizar.es

Correspondencia:

Alberto Carreras Gargallo

Departamento de Filosofía, Facultad de Filosofía y Letras

Cl. Pedro Cerbuna, 12 - 50009 ZARAGOZA

E-mail: acaras@unizar.es 